\title{
A Novel Wayfinding Service for Empowering Physical Activity
}

Conference Paper · October 2014

DOI: 10.13140/2.1.3218.6566

READS

38

4 authors, including:

Janice C Zgibor

University of South Florida

101 PUBLICATIONS 1,450 CITATIONS

SEE PROFILE

Monsak Socharoentum

National Electronics and Computer Techno...

11 PUBLICATIONS 6 CITATIONS

SEE PROFILE

\section{Gretchen Piatt}

University of Michigan

50 PUBLICATIONS 716 CITATIONS

SEE PROFILE 


\title{
A Novel Wayfinding Service for Empowering Physical Activity
}

\author{
Hassan A. Karimi ${ }^{1}$, Janice C. Zgibor $^{2}$, Gretchen A. Piatt ${ }^{3}$, Monsak Socharoentum ${ }^{1}$ \\ ${ }^{1}$ Geoinformatics Laboratory, School of Information Sciences, University of Pittsburgh \\ ${ }^{2}$ Center for Aging and Population Health-Prevention Research Center, University of \\ Pittsburgh \\ ${ }^{3}$ Department of Learning Health Science, School of Medicine Department of Health, \\ University of Michigan \\ \{hkarimi, edcjan, mos22\} @pitt.edu, piattg@med.umich.edu
}

\begin{abstract}
A wayfinding service for empowering physical activity is presented. The service finds routes that involve multi-modal transportation where walking is always one mode. The service is based on the new concept of multi-modal transportation with multi-criteria walking. A prototype of the service is developed and a new empowerment approach for it is discussed.
\end{abstract}

Keywords: physical activity, wayfinding service, walking paths

\section{Introduction}

As the obesity epidemic in the United States continues to worsen (Flegal et al. 2005), the health of communities is increasingly in jeopardy. Approximately $1 / 3$ of Americans are obese [Body Mass Index (BMI) $\geq 30 \mathrm{~kg} / \mathrm{m}^{2}$ ] and nearly $70 \%$ are considered overweight (BMI $\geq 25 \mathrm{~kg} / \mathrm{m}^{2}$ ). Since 1999 , a significant increase in BMI was observed for men with a borderline increase among women. These conditions substantially increase the risk for developing type 2 diabetes (diabetes) and/or cardiovascular disease and certain cancers (Pate et al. 1995). There is a significant body of evidence demonstrating the impact of physical activity on reducing body weight. As obesity is a significant risk factor for developing several chronic illnesses (Centers for Disease Control and Prevention 2013), there is a critical need to develop and implement practical, sustainable efforts that focus on increasing physical activity in "real world" community settings in order for patients, healthcare providers, and policy makers to make informed decisions regarding effective approaches to address the epidemic.

One of the most effective methods to combat obesity and prevent other chronic disease is increasing physical activity. Physical activity is an ideal intervention as it can be free or low cost and has demonstrated success. However, little progress is being made to increase physical activity in the United States (Carlson et al. 2010). Approximately $60 \%$ of Americans fail to achieve recommended physical activity goals with little progress noted over the past decade (Carlson et al. 2010). Current evidence-based physical activity guidelines recommend that adults age 18 and older 
get 2.5 hours of moderate intensity or 75 minutes of vigorous intensity aerobic physical activity per week (Centers for Disease Control and Prevention Division of Nutrition 2011). Despite this evidence, a host of barriers, including time, physical access, financial means, and confidence and enjoyment of the activity keep individuals from engaging in physical activity (Pate et al. 1995). Overcoming these barriers is challenging and short-term physical activity interventions, often, do not lead to long-term sustained physical activity behavior change, largely because of activity not completely being incorporated into day-to-day living. Additionally, successful maintenance of physical activity efforts is highly dependent on improving self-efficacy (or confidence) in a person's ability to be physically active while incorporating social support (Pate et al. 1995).

\section{Self-Efficacy}

Increasing individuals self-efficacy to engage in physical activity is a concept that is deeply rooted in Self-Determination Theory and self-motivation (Williams et al. 1998; Williams et al. 2004). An individual is more likely to be motivated (autonomy motivation) to develop the skills and capacity to self-regulate the behaviors needed to function effectively, if that individual views those behaviors as personally meaningful (Deci et al. 1994). In the context of physical activity, autonomy motivation refers to the extent to which individuals feel they are initiating and valuing specific physical activity behaviors and the type of approach they believe will lead to successful behavior change (Williams et al. 1998). Self-determination theory and autonomy support provide the framework for patient empowerment, which is defined as helping patients discover and develop the inherent capacity to be responsible for one's own life (Anderson et al. 1991). Empowerment plays a key role in enhancing the selfefficacy needed to engage in physical activity in the short and long term and is the theoretical basis for which we developed our prototype, Route2Health .

\section{Technology and Social Support}

The smart phone is a relatively novel platform through which to deliver empowerment-based physical activity information. It has the advantage of familiarity and flexibility, which are characteristics that may be particularly helpful to socioeconomically or geographically marginalized populations who may not have access to or little spare time to participate in traditional physical activity efforts.. Perhaps even more importantly, smart phones provide the capacity to offer an ecommunity for behavior reinforcement and social support. Online communities are very popular among people with chronic health conditions (Greene et al. 2011), as a source of information as well as emotional support and accountability that comes from connecting with others with similar conditions. (Hwang et al. 2010).

Despite the popularity of navigation services and the importance of physical activity, current navigation servicesare not designed to assist in improving or monitoring physical activity. Current navigation services operate in the uni-modal 
transportation mode (i.e., they provide only driving, riding public transportation, or walking) and do not take into account the factors that impact physical activity in finding routes. To fill in this gap, we present a new navigation service, called Route2health, which would be able to: (a) find a route with multi-modal transportation for each trip; (b) include walking always as one mode of transportation; and (c) take specific user's requirements for each walking session so that physical activity is personalized.

\section{Route2Health Foundation}

The foundation of Route2Helath, as a new service, is based on specific models and new algorithms, described in this section.

In the context of this paper, transportation refers to the traveling of people between locations by vehicles or on foot. Transportation can be classified into uni-modal, where only one mode of transportation (e.g., walking, driving) is involved or multimodal, where more than one mode of transportation (e.g., driving and walking) is involved. Trip refers to transportation from an origin to a destination. Trip can also be uni-modal or multi-modal. Path is a possible physical connection between origin and destination for the purpose of traversing by uni-modal or multi-modal transportation. There could be multiple possible paths for a trip, and travelers usually choose the optimal one, which is the best path with respect to one or more given criteria. Finding an optimal path requires a transportation network containing topological relationships of the real-world transportation infrastructures (e.g., road, bridge, tunnel, intersection, and sidewalk). Transportation networks are modelled as graphs where each node represents a location where travelers must make a traversing decision (e.g., turn left/right, get on/off vehicle, and switch between modes) and a link connects two nodes representing traversable passage (e.g., road segment and sidewalk segment). There is a value quantifying traversing cost associated with each link between its start and end nodes. Examples of traversing costs are distance, time, expense, air pollution, and slope. Transportation networks representing one mode of transportation are unimodal, and a multi-modal network is formed by combining different uni-modal networks with designated existing or new nodes or links for switching between them.

For calories estimation in Route2Health, we have considered the American College of Sports Medicine's (ACSM) ${ }^{1}$ work on amount of calories burned (energy expenditure) for several activities (e.g., walking, running, and stepping) which is formulated in an equation for walking. The ACSM walking equation (Tharrett and Peterson 2012) expresses walking energy expenditure as:

$$
E E=(0.1 \cdot S+1.8 \cdot S \cdot G+3.5) \cdot B M \cdot t \cdot 0.005
$$

\footnotetext{
${ }^{1}$ http://www.acsm.org/about-acsm/
} 
where $E E$ is walking energy expenditure (kilocalories); $S$ is walking speed (meter/minute); $G$ is grade (slope) in decimal form (e.g., 0.02 for $2 \%$ grade); BM is traveller's body weight (kg.); and tis walking time (minute). Equation (1) is based on the assumption that the traveller walks at a constant speed during time $t$, and the slope $G$ is homogeneous. The equation is only accurate for the speed between 60-100 meters per minute (Glass and Dwyer 2007).

Route2Health algorithm requires an origin, a destination, body weight, walking distance, and the desired mode of transportation (driving or riding). Walking transfer nodes, located within an acceptable walking distance, are retrieved and used for vehicular and walking path computation. In the absence of walking transfer nodes that satisfy the requested walking distance, the algorithm computes only walking paths that connect the origin and the destination. If walking transfer nodes (parking lots or bus stops) are found, the associated vehicular paths (driving or riding) are computed.

Once vehicular and walking paths are computed, the results (walking paths and vehicular paths) are combined to form multi-modal paths to link the origin, walking transfer nodes, and the destination. The number of walking transfer nodes determines the number of alternate walking paths. Then, based on Equation (1), the calorie burns for each alternate walking path is estimated. Slope of each segment of a walking path is estimated by using high-resolution Digital Elevation Model (DEM) data. Walking speed is provided by the user, or could be calculated based on walking path distance and estimated duration of walking. For each optimal path, path geometry, travel distance, travel time, and estimated calories burned are presented to the user. Figure 4 shows an example of a route computed for a walking-driving trip and Figure 5 shows an example of a route computed for a walking-riding trip.

\section{Empowering Physical Activity}

Theoretical frameworks are usually selected for use in program design and individual practice based on how well they meet one or more of the four purposes detailed below: describing, explaining, predicting, and controlling/influencing health behavior (Fain 1999; Meleis 2007; Fawcett 2013).

The first purpose of a theoretical framework is to describe the phenomena of interest, telling us the way things are, but not why they are the way they are or how they are likely to change. The second purpose of a theoretical framework is explanation. While a descriptive framework answers the question, "What is happening?", an explanatory framework answers the question of "Why is it happening?" The third purpose of a theoretical framework is prediction. Using data derived from other studies would support a prediction of similar results based on these factors. Finally, a theoretical framework is considered controlling or influencing when it has the ability to change behavior. The ability of a framework to influence achievement of greater levels of physical activity could be contrasted with a framework that is less useful because it cannot be manipulated to produce better outcomes. 


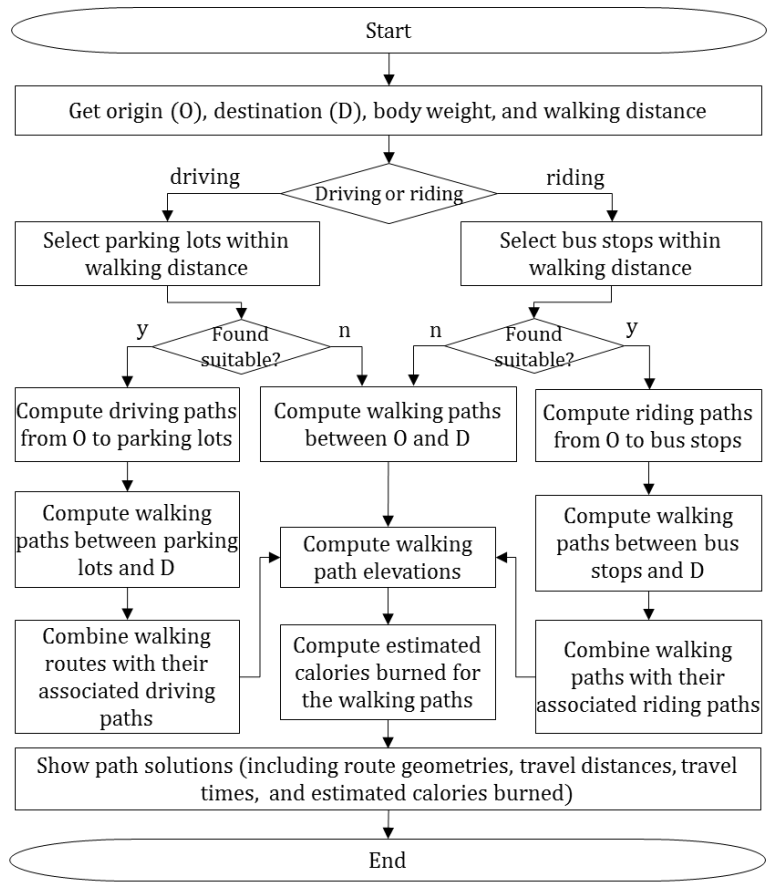

Fig. 3. Route2Health algorithm.

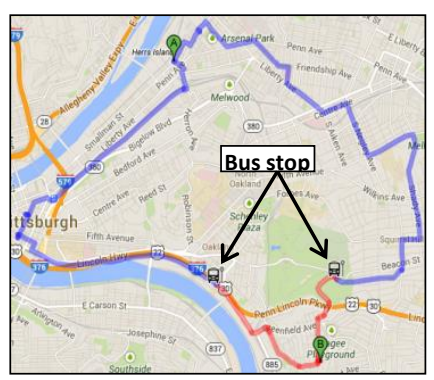

Fig. 4. Example walking-driving route.

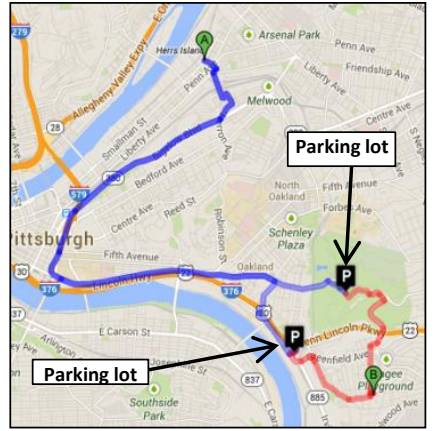

Fig. 5. Example walking-riding route.

The theory-based framework that we are using for Route2Health encompasses elements of the empowerment approach and self-determination theory and autonomy support (Deci et al. 1994) (Anderson et al. 1995; Williams et al. 1998; Funnell and Anderson 2003; Funnell and Anderson 2004; Williams et al. 2004; Anderson et al. 2009). These elements were combined into a theoretical framework for this project as they complement each other and are proven constructs in successful health behavior interventions. The chosen elements combine strategies for management of the social and behavioral factors associated with physical activity behavior change. Figure 6 depicts the theory-based framework.

\section{Team-Based Empowerment for Social Support}

An innovative aspect of Route2Health is the use of social media to form teams. Team identification will be encouraged through a process of team naming and an inter-team walking competition to create a collective outcome. Individuals will be able to see not only their own individually tailored messaging and feedback about their walking patterns, but also the percent of their team members who have achieved their physical activity goals for that day and what cumulative percent of goals the team achieved over the month. They will also see this same information for other teams.

We believe that assignment to teams may encourage physical activity behavior change. "Team" behavior differs from "group" behavior in that outcomes are assessed for the team as well as for the individual, and the former is contingent on the latter; in 
small studies, assignment to teams rather than groups has promoted adherence (Carter 2011). Teams can create camaraderie and accountability. In addition, seeing that most other members of one's team are walking can create a "descriptive norm" that will lead others who are not walking as much to walk more often (Krupka, 2009). In a correlational study of a large physical activity program where people voluntarily chose whether to join as part of a team, those who did so continued reporting their activity for longer and reported higher levels of activity when they did so (Richardson et al. 2007; Richardson et al. 2008).

Social media is a novel platform through which to incorporate and study behavior change. Evidence demonstrates that the Internet can be effectively used for weight loss interventions and can be useful in weight loss maintenance (Arem and Irwin 2011) as it has the advantage of familiarity and flexibility. ,Interviews with users of SparkPeople revealed that some felt that interactions with people on SparkPeople were more helpful than those with real-life friends and family. (Hwang et al. 2010). Evidence also demonstrates that an online community may increase motivation and adherence to online pedometer interventions, potentially through mechanisms above as well as through friendly competitions (Resnick et al. 2010; Richardson et al. 2010). (Consolvo et al. 2006). Route2Health has the potential to build on this above evidence by incorporating physical activity into daily routines, an integral component of making and maintaining physical activity behavior change..

To facilitate team-based empowerment, SoNavNet (Karimi et al., 2009a; Karimi et al., 2009b), a social navigation networking system, will be used. Through SoNavNet, Route2Helath searches for possible recommendations, in particular recommend routes that are, otherwise, not easy to compute, due to lack of modeling and quantifying multiple criteria. An example of how SoNavNet can be used in Route2Health is when a member shares his/her experience about a trip where he/she used a car to drive to a parking lot and walked to and from a destination. In this example, the walking component of the route will be rated by using specific physical activity parameters.

Through the SoNavNet component of Route2Health, specific physical activity parameters of a route can be rated, all possible walking-driving or walking-riding options between origin and destination locations can be searched, and the most optimal (personalized) walking component from among all possible options to find the closest match (recommended by other members of SoNavNet) to a given set of user's criteria can be found.

\section{Prototype}

A prototype Route2Health has been developed. Figure 7 shows the architecture of the Route2Health application. The architecture is composed of six components: (1) Walking Transfer Selector, (2) Vehicular Path Alternative Generator, (3) Walking Path Alternative Generator, (4) Path Combiner, (5) Objective Function Normalizer, and (6) Multi-Criteria Optimizer. Walking Transfer Selector is the component that collects inputs: origin, destination, and personal walking distance limit. Based on the inputs, Walking Transfer Selector would identify all possible feasible walking transfers. Then based on the origin, the destination, and the identified walking 
transfers, the relevant walking path alternatives and vehicular path alternatives are computed by Walking Path Alternative Generator and Vehicular Path Alternative Generator, respectively. The path alternatives of the two modes are then combined into a complete path alternative by the Path Combiner. In the Objective Function Normalizer, the path alternatives are quantified using objective functions, and the outputs from the objective functions are normalized. The normalized values are then optimized by the Multi-Criteria Optimizer to obtain the final solution (optimal path).

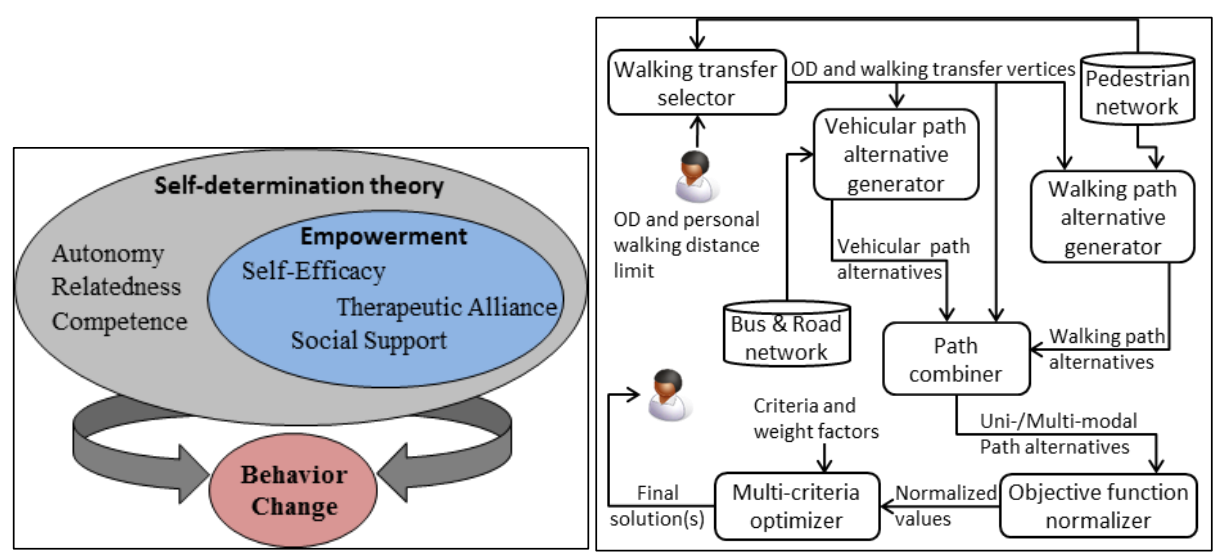

Fig. 6. Theory-based Conceptual Framework.

Fig. 7. Route2Health architecture.

\section{Summary}

A new service, called Route2Health, is presented where it can be used to empower people in finding optimal routes to improve physical activity. The service is based on the assumption that for any trip there should be a walking component. The MMTMCW concept is introduced which can find routes that involve multi-modal transportation and find most optimal walking path among the candidate walking paths.

\section{References}

1. Anderson, R. M., M. M. Funnell, et al. (2009). "Evaluating the Efficacy of an Empowerment-Based Self-Management Consultant Intervention: Results of a Two-Year Randomized Controlled Trial." Therapeutic Patient Education 1(1): 3-11.

2. Anderson, R. M., M. M. Funnell, et al. (1991). "Learning to empower patients." Diabetes Care 14(7): 584-590.

3. Anderson, R. M., M. M. Funnell, et al. (1995). "Patient empowerment: results of a randomized control trial." Diabetes Care 18: 943-949.

4. Arem, H. and M. Irwin (2011). "A review of web-based weight loss interventions in adults." Obes Rev 15(5): 3236-3243. 
5. Carlson, S. A., J. E. Fulton, et al. (2010). "Trend and prevalence estimates based on the 2008 Physical Activity Guidelines for Americans." Am J Prev Med 39(4): 305-313.

6. Centers for Disease Control and Prevention. (2013). Retrieved December 3, 2013, from (http://www.cdc.gov/obesity/data/trends.html\#State). .

7. Centers for Disease Control and Prevention. (2013). "Obesity: Halting the Epidemic by Making Health Easier At A Glance 2011." Retrieved December 3, 2013, from http://www.cdc.gov/chronicdisease/resources/publications/AAG/obesity.htm.

8. Centers for Disease Control and Prevention Division of Nutrition, P. A., and Obesity, National Center for Chronic Disease Prevention and Health Promotion. (2011, December 1, 2011). "Physical Activity for Everyone." Retrieved January 13, 2011, from http://www.cdc.gov/physicalactivity/everyone/guidelines/adults.html.

9. Consolvo, S., K. Everitt, et al. (2006). "Design requirements for technologies that encourage physical activity." CHI.

10. Deci, E. L., H. Eghrari, et al. (1994). "Facilitating internalization: The self-determination theory perspective." Journal of Personality 62: 119-142.

11. Flegal, K. M., B. I. Graubard, et al. (2005). "Excess deaths associated with underweight, overweight, and obesity." JAMA 293(15): 1861-1867.

12. Funnell, M. M. and R. M. Anderson (2003). "Patient Empowerment: a look back, a look ahead." Diabetes Educator 29(3): 454-460.

13. Funnell, M. M. and R. M. Anderson (2004). "Empowerment and Self-Management of Diabetes. Clinical Diabetes." Clinical Diabetes 22(3): 123-127.

14. Glass, S., and Dwyer, G. B. (Eds.). (2007). ACSM's Metabolic Calculations Handbook. Lippincott Williams \& Wilkins, 19 \& 26.

15. Greene, J., N. Choudhry, et al. (2011). "Online social networking by patients with diabetes: a qualitative evaluation of communication with Facebook." J Gen Intern Med 26(3): 287-292.

16. Hwang, K., A. Ottenbacher, et al. (2010). "Social support in an internet weight-loss community." Int J Med Inform 79(1): 5-13.

17. Karimi, H. A. Zimmerman, B. Ozcelik, A. and Roongpiboonsopit, D. SoNavNet: A Framework for Social Navigation Networks. In International Workshop on Location Based Social Networks (LBSN'09). Seattle, WA, November 3-6 (2009).

18. Pate, R. R., M. Pratt, et al. (1995). "Physical activity and public health. A recommendation from the Centers for Disease Control and Prevention and the American College of Sports Medicine." JAMA 273(5): 402-407.

19. Resnick, P., A. Janney, et al. (2010). "Adding an online community to an internetmediated walking program. Part 2: strategies for encouraging community participation." Med Internet Res 12(4): e72.

20. Richardson, C., L. Buis, et al. (2010). "An online community improves adherence in an internet-mediated walking program. Part 1: results of a randomized controlled trial." J Med Internet Res 12(4): e71.

21. Richardson, C., K. Mehari, et al. (2007). "A randomized trial comparing structured and lifestyle goals in an internet-mediated walking program for people with type 2 diabetes." Int J Behav Nutr Phys Act 16(4): 59.

22. Richardson, C., T. Newton, et al. (2008). "A meta-analysis of pedometer-based walking interventions and weight loss." Ann Fam Med 1: 69-77.

23. Tharrett, S. J., and Peterson, J. A. (Eds.). (2012). ACSM's health/fitness facility standards and guidelines. Human Kinetics

24. Williams, G. C., Z. R. Freedman, et al. (1998). "Supporting autonomy to motivate patients with diabetes for glucose control." Diabetes Care 21(10): 1644-1651.

25. Williams, G. C., H. A. McGregor, et al. (2004). "Testing a self-determination theory process model for promoting glycemic control through diabetes self-management." Health Psychology 23(1): 58-66. 
